\title{
Eight year experience in open ended instrumentation laboratory
}

\section{Manuel Marques, Carla Rosa, Paulo V. Marques}

Manuel B. Marques, Carla C. Rosa, Paulo V. S. Marques, "Eight year experience in open ended instrumentation laboratory," Proc. SPIE 9793, Education and Training in Optics and Photonics: ETOP 2015, 979327 (8 October 2015); doi: 10.1117/12.2223201

SPIE Event: Education and Training in Optics and Photonics: ETOP 2015, 2015, Bordeaux, France 


\title{
Eight year experience in open ended instrumentation laboratory Manuel B. Marques*, Carla C. Rosa, Paulo V. S. Marques \\ Dep. Física e Astronomia - FCUP, and INESC TEC \\ Rua do Campo Alegre s/n, 4169-007 PORTO, Portugal
}

\begin{abstract}
When designing laboratory courses in a Physics Major we consider a range of objectives: teaching Physics; developing lab competencies; instrument control and data acquisition; learning about measurement errors and error propagation; an introduction to project management; team work skills and scientific writing. But nowadays we face pressure to decrease laboratory hours due to the cost involved. Many universities are replacing lab classes for simulation activities, hiring $\mathrm{PhD}$. and master students to give first year lab classes, and reducing lab hours. This leads to formatted lab scripts and poor autonomy of the students, and failure to enhance creativity and autonomy.

In this paper we present our eight year experience with a laboratory course that is mandatory in the third year of Physics and Physical Engineering degrees. Since the students had previously two standard laboratory courses, we focused on teaching instrumentation and giving students autonomy. The course is divided in two parts: one third is dedicated to learn computer controlled instrumentation and data acquisition (based in LabView); the final 2/3 is dedicated to a group project. In this project, the team ( 2 or 3 students) must develop a project and present it in a typical conference format at the end of the semester. The project assignments are usually not very detailed (about two or three lines long), giving only general guidelines pointing to a successful project (students often recycle objectives putting forward a very personal project); all of them require assembling some hardware. Due to our background, about one third of the projects are related to Optics.
\end{abstract}

Keywords: Laboratory teaching, LabView, Open ended project

\section{INTRODUCTION}

Most laboratory courses in Science degrees are based on the demonstration of a law or effect, or on teaching a specific technique. There isn't usually any focus on the development of problem solving competencies. The idea of open ended projects is left to the end of the degree, typically to the master degree dissertation. But, by then, most students take a project that is well defined, avoiding the ones that don't ensure an easy completion within the allowed time frame. This is understandable, as students are already looking for employment or grants.

The learning experience of a completely open ended project, where the students don't know if the first idea of the project can be completed during the semester, is very important to their development. Relocating this type of project to the bachelor degree removes all the fuss about finishing the degree in time, and allows work with simpler problems than those that should be tackled in a master degree.

The course, in this format, was established during the reformulation to meet the Bologna Process objectives. The design was based on the teachers' expertise in student orientation of Ph. D. and M. Sc. work and of grantees in industry oriented projects.

The students are challenged to develop a project of instrumentation and automation of scientific experiments. The project begins with a conceptual idea, ending with the project building, testing and demonstration of the associated prototype. The project is presented in a report and orally in a small conference.

We have chosen LabVIEW ${ }^{1}$ as the software to be used in the course, based on our experience and its easy learning. LabVIEW software and National Instruments interfaces are expensive when compared to other resources available. Arduino $^{2}$ interfaces with sensors can be acquired for less than 100 euro and the software to control it is cheap or freeware. But, in our opinion, although using Arduino with a few sensors is easily achieved by a student with some familiarity with programming, communicating with an instrument through its IEEE 488 interface (GPIB) or USB interface is beyond the scope of the course. The neat approach of LabVIEW ${ }^{3,4}$ to communication with instruments through its virtual interface (VISA - Virtual Instrument Software Architecture), allowing communication and control of

*mbmarque@fc.up.pt; phone +351 220402301; fax +351 220402437

Education and Training in Optics and Photonics: ETOP 2015, edited by Eric Cormier, Laurent Sarger Proc. of SPIE Vol. 9793, 979327 · @ 2015 SPIE, IEEE, OSA, ICO · doi: 10.1117/12.2223201 
an instrument independently of the type of interface used, and the simplicity of graphical programming, were fundamental for our choice.

\section{COURSE DESIGN}

Designing the course focused on the type of instrumentation to be studied and used by the students. It seemed relevant to develop a broad structure allowing for the integration and application of knowledge in Electronics, Signals and Systems and Signal Processing, as well as in instrument control and automatic data acquisition. These competencies are looked for and prized in this specific labour market.

In order to encourage students, we adopted an approach inspired by Problem Solving. The proposed model requires a readiness for work that is similar to the one required in the business/industrial world and stimulates the development of important capacities for professional insertion, namely team work, autonomy, research and technical dialogue with electronic/mechanical workshops staff, as well as the development of communication capabilities in written reports and oral presentations.

As a second motivation, it is intended that the students go through a different reality, away from what they have been exposed to in previous experimental classes, and near the daily reality of a researcher/technician in his professional activities. The Problem Solving model moves away from the student passivity in the more traditional laboratory classes where many directed experimental protocols are used, limiting creativity and capacity of overall analysis. The initial disruption is rapidly transformed into a source of motivation and an increase in the ability to overcome obstacles and technical difficulties.

\section{Methodologies}

We want students to be able to solve a specific problem posed by scientific instrumentation, using problem solving methodology. This includes the definition of the work plan, separating the accessory from the fundamental, and, starting from scratch, the development, testing and demonstration of the final solution.

The strategy was planned around the competencies related to knowledge and control of instrumentation that can be acquired in a third year bachelor course with 7.5 ECTS. In the two previous years, the students took the fundamental courses on Mathematics and Physics, as well as two classical laboratory courses.

In planning the course, the instrumentation was chosen in order to have a broad spectrum of instruments, including old GPIB controlled instruments that are still present in many laboratories, as well as new USB controlled instruments. Students were also required to do the drawing of mechanical parts and electronic circuits to be built at the Department's mechanical and electronic workshops, and to come up with innovative solutions.

The final report allows the evaluation of the student's capacities of synthesis and analysis. Teachers are aware that a group report may lead to incompatibilities in organization and style; to prevent this, they undertake a close supervision looking for signals of emerging problems, in order to take action before it escalates.

The oral presentation, made by each working group, is an essential evaluation tool. It gives students the understanding that it is very important to learn how to communicate and present, clearly and briefly, the strategy followed and the results obtained, as well as a critical retrospective of the decisions taken. The format adopted is similar to that in scientific conferences: 12 minutes of presentation followed by 8 minutes of questions. The two elements of the group must participate in the presentation and answer the questions, giving this component of evaluation the ability to distinguish between the group elements. All students are required to be present at all presentations and encouraged to ask their colleagues questions. This session is public for the whole staff of the department.

\section{Spaces}

Classes take place in a $150 \mathrm{~m}^{2}$ laboratory that is separated in two sections. The larger one is dedicated to Electronics, having ten working benches fitted with basic equipment and four tables for support. The section dedicated to instrumentation has ten workplaces equipped with computers, and four for support. The students work in groups of two and classes have a maximum of 16 students. The instrumentation laboratory is equipped with multiple acquisition interfaces (from several manufacturers), digital oscilloscopes, power supplies, signal generators and multimeters, all allowing computer control. Students can use the Electronics laboratory to construct small setups and have the supports of the department workshops to build small components for the project. 


\section{STUDENT PROJECTS IN OPTICS}

Due to the scientific background of the teachers involved, a large number of projects are related to Optics. A sample of the projects in optics from the last years is presented next.

\section{Photoelectric effect and measurement of $h$}

It was proposed to the students to fully automate an experiment to measure the stopping potential as a function of wavelength and, from those potentials, to calculate the value of the Planck constant. It was proposed to use several LEDs instead of the traditional Mercury lamp and spectral filtering.

The students studied the problem, with some guidance from teachers, and divided it into several small problems: lighting one LED at a time (interface, software and transistor circuits), measuring the stopping potential (capacitor charged to the potential, communicating with the voltmeter through the GPIB interface), passing light from the LEDs to the cell, reducing ambient light and averaging and storing data in the computer for posterior analysis. After testing each subset, everything was assembled together and tested again before the final measurements. The final assembly is presented in figure 1.

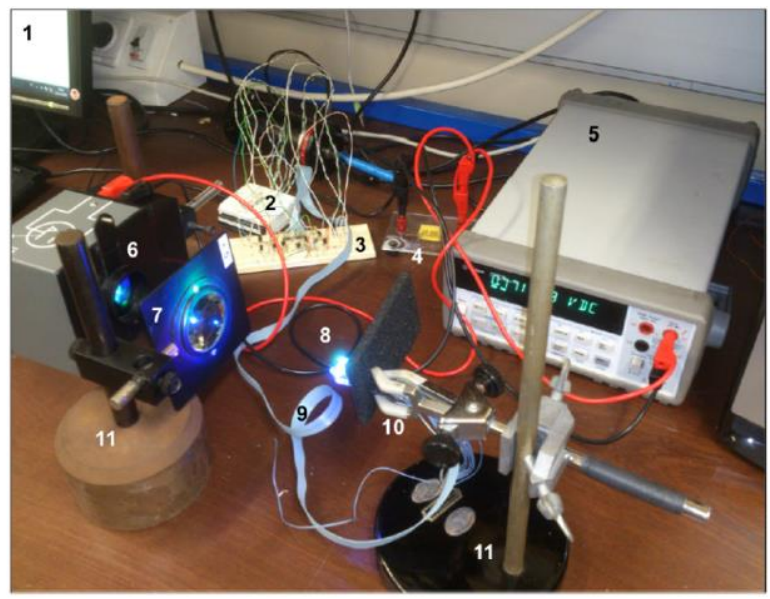

Figure 1. Student setup for the photoelectric effect. 1- Computer; 2- Interface (USB-6009); 3- Electronic control circuit; 4Capacitor (100 nF); 5- Voltmeter (not needed); 6- Photoelectric cell (Phywe); 7- lens; 8- LEDs; 9- Cable for electric connection of LEDs; 10 and 11- Mechanical holders.

With this setup, students obtained automatically an averaged value for $\mathrm{h}=5.110^{-34} \mathrm{~J} \mathrm{~s}$ and an extraction energy of 1.43 $\mathrm{eV}$. In figure 2 data averaged for each LED is presented with error bars.

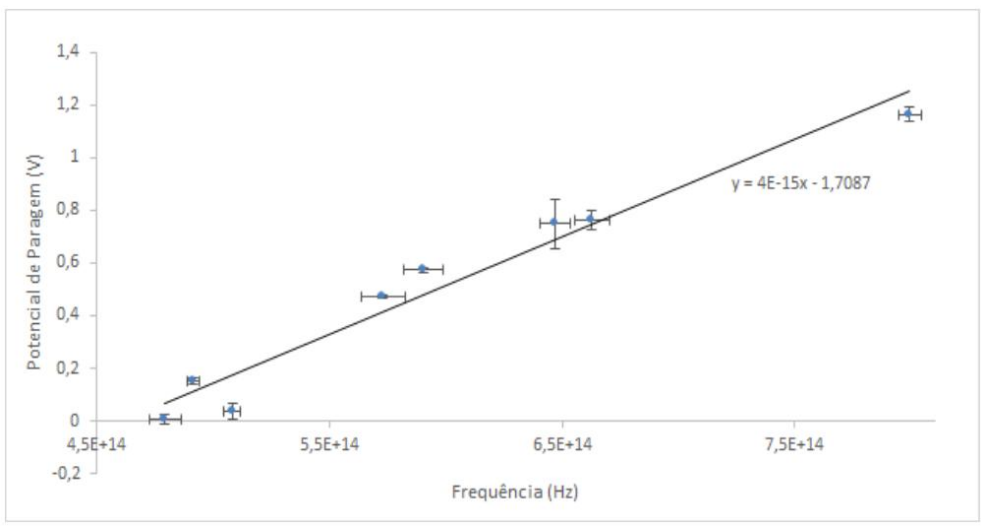

Figure 2. Student averaged results for the photoelectric effect. Horizontal error bars are based on the LEDs' spectral width and the vertical ones are the variance on the stopping potential.

Students weren't very satisfied with the large difference to the accepted value, but when they were driven towards a deeper analysis of the LED spectra, they noticed that the low power skirt was very different among the LEDs, 
particularly on the short wavelength side (this is the trade off to simplify the experiment without the use of spectral lamps). The students got convinced of this fundamental flaw by removing the two worst LEDs, thereby improving the value of $\mathrm{h}$ to $6.410^{-34} \mathrm{~J} \mathrm{~s}$.

\section{Acquiring diffraction patterns with one photodiode and a rotating mirror}

This proposition was to take a stepper motor (from an old photocopier), an interface, a mirror and a photodiode and record the diffraction pattern to the computer. After dealing with the electronics and four digital outputs from the interface for control, the students discovered that the step angle was larger than the angular diffraction pattern. Teachers were about to give then a thinner diffraction object when they came up with the idea of using reducing gears to get a smaller angular step, building it with the set of LEGO Technic available in the laboratory. Figure 3 presents the schematics of the setup and a photo of the gear detail and figure 4 shows the result obtained with a hair from a student.

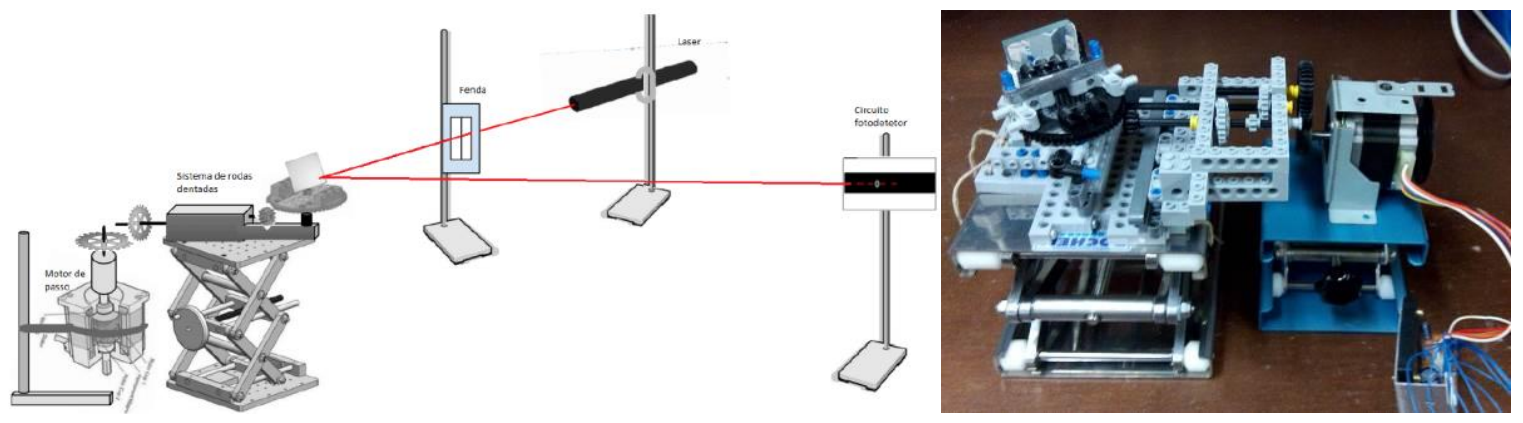

Figure 3. Student setup for the measurement of diffraction patterns.

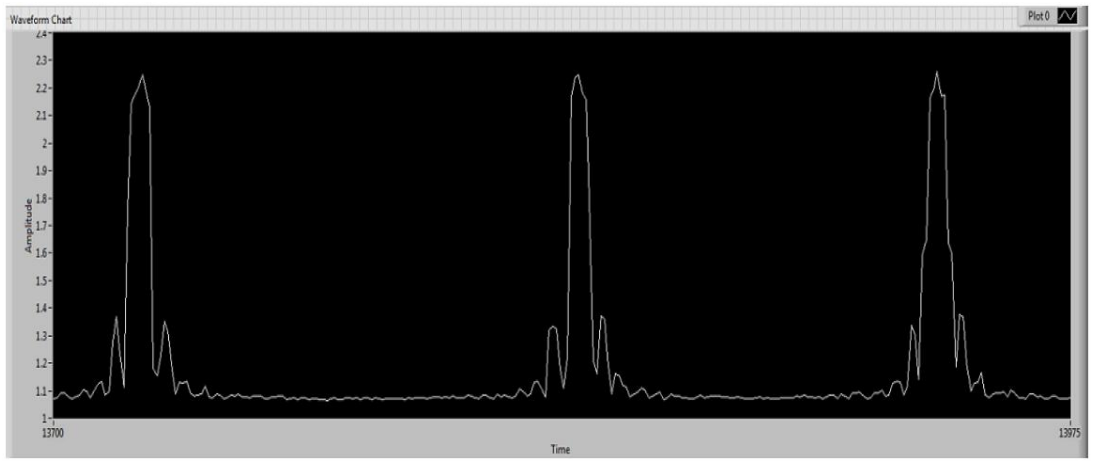

Figure 4. Diffraction pattern of a hair obtained with the setup.

\section{Acquiring fluorescence spectra and its variation with temperature}

The problem was to acquire fluorescence spectra as a function of temperature. Partial problems: controlling a mini USB spectrometer with optical fiber input, turning on and off a violet LED, acquire temperature from a thermistor and storing and processing spectra. In figure 5 we present the students' solution for the experimental setup. Some experimental results are presented in figure 6. 


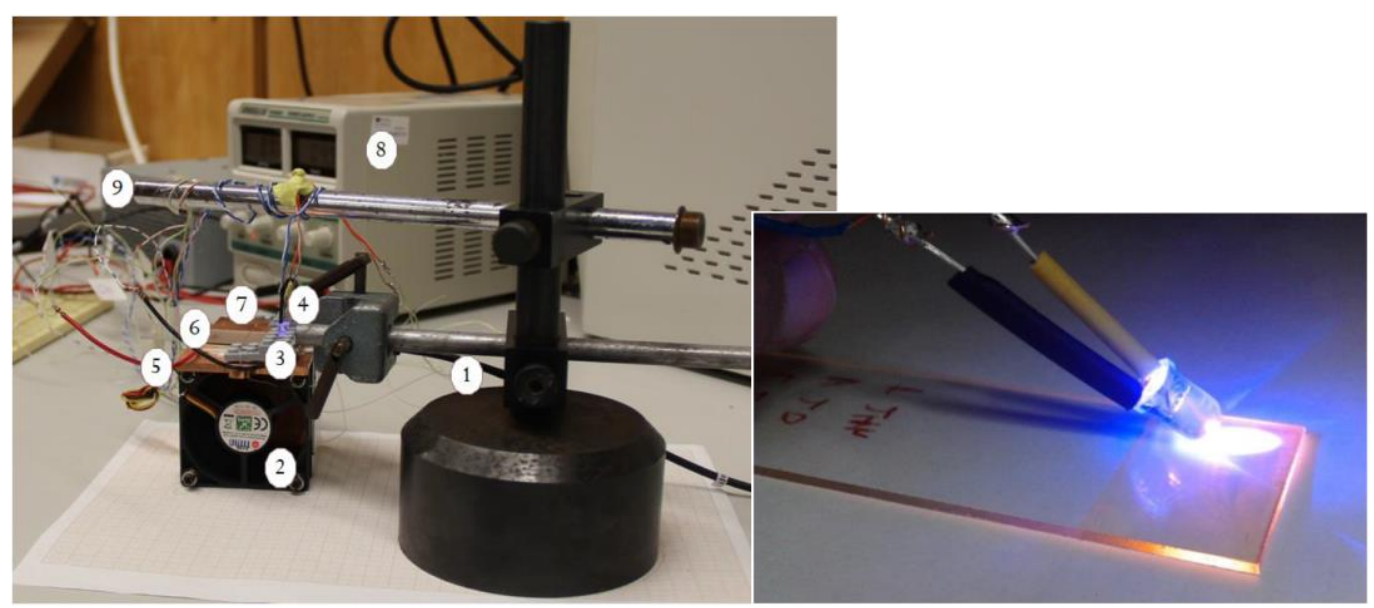

Figure 5. Setup for fluorescence measurements.
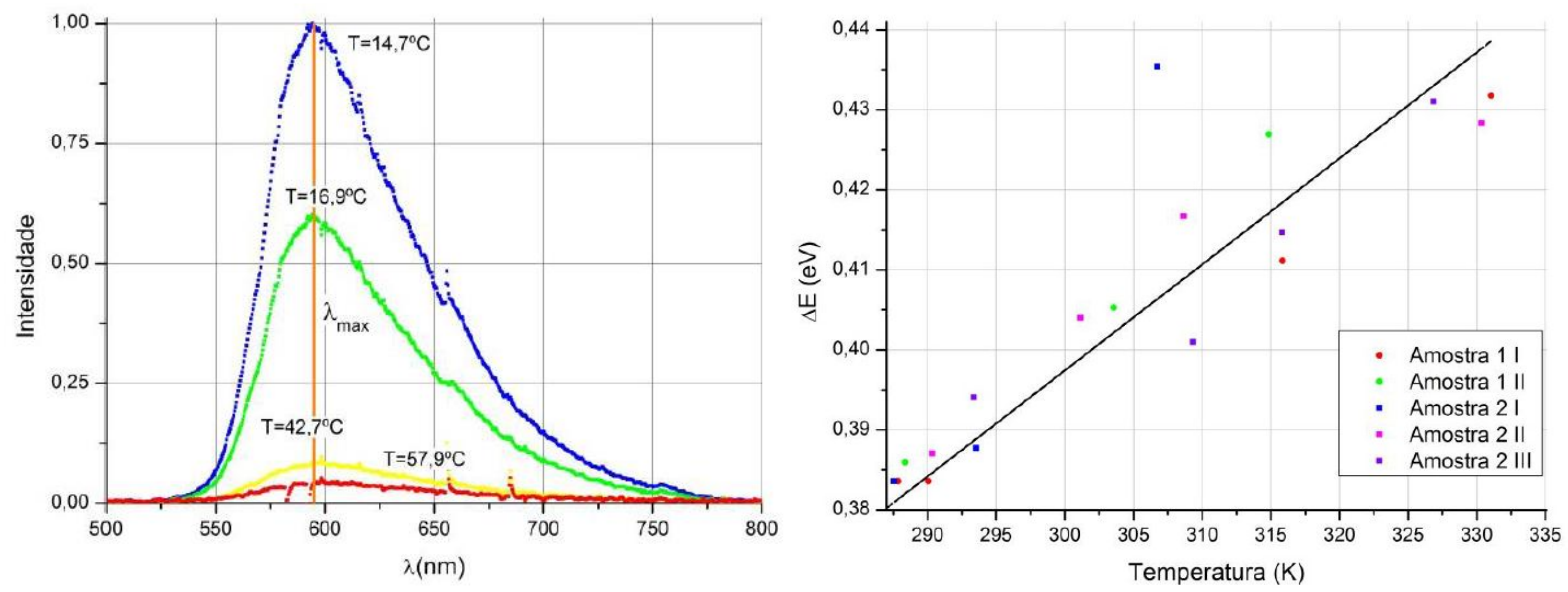

Figure 6. Left- Fluorescence spectra as a function of temperature. Right- Energy difference $(\Delta \mathrm{E})$ associated with spectrum width as a function of temperature.

\section{Other projects}

Some projects are proposed by research groups in activity in the Physics and Astronomy Department. They are presented to the students after an evaluation of the workload and level of knowledge needed is considered acceptable for them. Those projects need a special attention, in order to keep the workload within the limits established for the course. Two examples in recent years have been the control of the translation stages for a fs direct writing setup and the automatic data acquisition and partial control in a magneto-optical setup.

Other projects are proposed that seem easier than average (examples: simulation of traffic lights and speed trap and automatic control of the illumination using a photo resistor as the detector). These projects can be very interesting since students with low self-esteem can easily complete the more basic part of the project and feel able to build other activities from there.

\section{CONCLUSIONS}

The academic results confirm the excellent involvement of the students in the course. The students come spontaneously to the laboratory during its open hours and ask for more time in the laboratory.

After the first impact, the students begin to realize that they are capable of developing a project which they seriously doubted they were able to cope with; very quickly the project becomes a strong source of motivation. 
The approval rate and the grades attained demonstrate this extra motivation from the students. With a few exceptions, all students succeed in the course (the approval percentage is significantly higher than in previous laboratory courses) with grades that are higher than their average scores.

The major achievements of this course are:

- The students are stimulated by the course designed using Problem Solving-related strategies.

- The development of competencies in instrumentation and problem solving is an employability factor for the students.

- The great diversity of projects undertaken by the students gives them a wide range of application examples.

There are a few threats to this course. We identified two major ones:

- This course is critically dependent on the three authors' involvement and heavily on strong interaction with students in the first weeks of the duration of the project. More than one teacher is required in class, and usually we participate even when we are not allocated to the course. Most younger people from the teaching staff are more concerned with research activities, for professional progression, and are not in the mood to spend a lot of time with students, apart from which is mandatory.

- There is a great tendency of the students for programming, undervaluing the competencies in the understanding of the physical processes and in the knowledge of instrumentation and signal processing.

\section{Acknowledgements}

This work is partially financed by the FCT - Fundação para a Ciência e a Tecnologia (Portuguese Foundation for Science and Technology) within project UID/EEA/50014/2013.

\section{REFERENCES}

[1] National Instruments - LabVIEW, http://www.ni.com/labview

[2] Arduino, http://www.arduino.cc/

[3] Lauterburg, U., "LabVIEW ${ }^{\mathrm{TM}}$ in Physics Education", University of Bern, Switzerland, 2004, (22 May 2015) http://tycphysics.org/FL03_Sm04/LVLPMtS11_03/LV-PhysicsWPScreen.pd .

[4] Garg, A., Sharma, R., Dhingr, V., "Development of an Automated Modern Undergraduate Optics Laboratory using LabVIEW", The Education and Training in Optics and Photonics Conference - ETOP2009, 7.5.48, (2009). 